\title{
Retos para la vida independiente de las personas con discapacidad intelectual. Un estudio basado en sus opiniones, las de sus familias y las de los profesionales
}

Challenges for independent living of people with intellectual disabilities. A study based on their opinions, opinions of their families and professionals

\begin{abstract}
Resumen
A pesar de la ratificación de España de la Convención sobre los Derechos de las Personas con Discapacidad en 2007, son escasos los estudios sobre la situación de las personas con discapacidad intelectual (DI) respecto a su derecho a la vida independiente (art. 19). Con el objetivo de analizar las barreras, los apoyos y los retos que inciden en el ejercicio de este derecho, se realizó un estudio cualitativo con el que se aplicaron 10 grupos focales y 22 entrevistas individuales a personas con discapacidad intelectual, 5 grupos focales con familias y 33 entrevistas individuales con profesionales. Los resultados de la investigación ponen de manifiesto la necesidad de garantizar la universalidad del derecho a la vida independiente, ofrecer apoyos personalizados de calidad y desarrollar acciones formativas y de sensibilización en materia de derechos con los distintos actores implicados.
\end{abstract}

\section{Palabras clave}

Vida independiente, discapacidad intelectual, inclusión social, políticas sociales, apoyos personalizados.

\begin{abstract}
Despite Spain's ratification of the Convention on the Rights of Persons with Disabilities in 2007, there are few studies on the situation of people with intellectual disabilities (ID) regarding their right to independent living (art. 19). In order to analyse the barriers, supports and challenges that affect the exercise of this right, a qualitative study was developed through 10 focus groups and 22 individual interviews with people with ID, 5 focus groups with families, and 33 individual interviews with professionals. The research results show the need to guarantee the universality of the right to independent living, offering quality personalized supports and developing training and awareness actions in the area of rights with the different actors involved.
\end{abstract}

\section{Keywords}

Independent living, intellectual disability, social inclusion, social policies, personalized supports.

\author{
María Pallisera Díaz \\ $<$ maria.pallisera@udg.edu> \\ Universidad de Girona. España \\ Judit Fullana Noell \\ $<$ judit.fullana@udg.edu> \\ Universidad de Girona. España
}

Carol Puyaltó Rovira <carol.puyalto.rovira@umoncton.ca> Université de Moncton. Canadá

\section{Montserrat Vilà Suñé}

<montserrat.vila@udg.edu>

Universidad de Girona. España

María Josep Valls Gabernet <pepavalls@pip.udl.cat> Universidad de Lleida. España

\section{Gemma Díaz Garolera} <gemma.diaz@udg.edu> Universidad de Girona. España

Montse Castro Belmonte <montse.castro@udg.edu>

Universidad de Girona. España

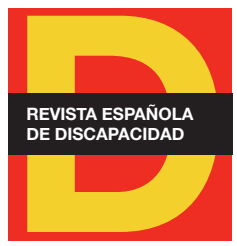

Para citar:

Pallisera, M. et al. (2018): "Retos para la vida independiente de las personas con discapacidad intelectual. Un estudio basado en sus opiniones, las de sus familias y las de los profesionales". Revista Española de Discapacidad, 6 (I): 7-29.

Doi: <https://doi.org/10.5569/23405104.06.01.01>

Fecha de recepción: 24-07-2017 Fecha de aceptación: 12-03-2018 


\section{Introducción. Modelos de apoyo a la vida independiente: breve evolución y situación actual}

La Convención sobre los Derechos de las Personas con Discapacidad (CDPD) (Naciones Unidas, 2006) ha supuesto el reconocimiento en el ámbito internacional de la persona con discapacidad como sujeto de derechos. Constituye una norma vinculante para los países que la han ratificado (España lo hace el 23 de noviembre de 2007, entrando en vigor el 3 de mayo de 2008). Dicha ratificación compromete a los países a garantizar que las personas con discapacidad puedan participar plenamente en la sociedad, tomen decisiones sobre sus vidas y reciban los apoyos necesarios para gozar plenamente de los derechos vinculados con su inclusión social (educación, accesibilidad, vida independiente, capacidad de obrar, etc.). La CDPD es, en estos momentos, una herramienta clave para avanzar tanto en el diseño como en la evaluación de las políticas y en las prácticas sociales y educativas, puesto que orienta hacia los objetivos a conseguir para la plena inclusión y plantea argumentos para que las personas con discapacidad puedan defender y reivindicar sus derechos. Asimismo, constituye un modelo a partir del cual realizar diagnósticos, evaluar logros de las políticas y prácticas socioeducativas, y plantear propuestas de mejora (Shogren y Turnbull, 2014).

Centrándonos en la vida independiente (VI), el artículo 19 de la Convención establece el derecho de todas las personas con discapacidad a decidir dónde quieren vivir y con quién, y a acceder a los apoyos que les permitan igualdad de acceso a los recursos de la comunidad. Ello supone cuestionar la tendencia generalizada de atención residencial a las personas con discapacidad intelectual, dominante en los países occidentales desde la segunda mitad del siglo XX (Emerson y Ramcharan, 2010). Es en los años 70 y 80, en los EEUU, Canadá, Reino Unido y Australia, entre otros, en los que se desarrolla un creciente movimiento de crítica a este modelo, basado en evidencias de conculcación de derechos de las personas atendidas (Barnes y Mercer, 2010; Kilbane y McLean, 2008), así como de despersonalización y exclusión social (European Commission, 2009; Mansell et al., 2010). Estas críticas conducen a un nuevo modelo de apoyo a la vida independiente basado en la comunidad. Se pretende que las personas con discapacidad puedan vivir siguiendo los patrones utilizados por la mayoría de la población en el contexto donde se desarrollan, con una buena calidad de vida y participando como ciudadanos de pleno derecho en las actividades económicas, sociales y culturales de acuerdo con sus capacidades y deseos (Mansell et al., 2010).

En estos momentos, la tendencia generalizada en la mayoría de los países occidentales es la transición desde un modelo residencial (congregated settings) a un modelo basado en viviendas más pequeñas (group homes o hogares de grupo) y al apoyo personalizado (personalized living settings) (Wahlström et al., 2014). En los hogares de grupo (también llamados viviendas tuteladas), un grupo de entre 3 a 10 personas (aproximadamente) comparten vivienda con el apoyo de profesionales durante toda la jornada. La constatación de ciertas limitaciones que conllevan estas viviendas (falta de privacidad, dificultades para poder tomar decisiones, etc.) (Hollomotz y The Speakup Commitee, 2009; García-Iriarte et al., 2016) ha llevado a desarrollar un modelo de apoyo personalizado a la vida independiente. Se trata de ofrecer apoyos individuales a una persona que vive sola o en pareja o comparte piso con una o dos personas de acuerdo con sus necesidades y preferencias (McConkey et al., 2016). La implementación de este modelo, vinculado directamente al desarrollo de prácticas y políticas que potencian la elección personal, avanza muy lentamente (García Iriarte et al., 2016).

En España, la atención a las personas con DI se ha gestionado a partir de la Ley de Integración Social del minusválido (LISMI, 1982) combinando la atención institucional con los servicios basados en la comunidad, 
dominando en este último caso el modelo de pisos/viviendas tuteladas. En el año 2006 se aprueba la Ley de promoción de la autonomía personal y atención a las personas en situación de dependencia que establece, entre sus prestaciones, la figura del asistente personal. Ahora bien, a pesar de la década transcurrida, la implantación hoy de esta figura es todavía muy incipiente y con escasa participación en términos cuantitativos (Huete y Quezada, 201411). Además, esta figura se ha vinculado a la promoción de la autonomía de las personas con gran dependencia, por lo que las personas con menor grado de dependencia, entre las que se incluyen la mayoría de las que tienen DI, no pueden beneficiarse de ella. La Ley General de derechos de las personas con discapacidad y de su inclusión social (2013), que sustituye a la LISMI (1982), estructura su articulado teniendo en cuenta la Convención de las Naciones Unidas, y reconoce la vida independiente (VI) como uno de sus principios (art. 3), dedicándole un capítulo. No obstante, aunque esta ley plantea la necesidad de establecer condiciones básicas de accesibilidad y no discriminación en la comunidad, no se llega a la concreción del artículo 19 de la convención y se reproducen, en su artículo 51, los servicios establecidos por la LISMI.

En distintas comunidades autónomas del estado español existen algunos proyectos específicos de apoyo a la vida independiente en el propio hogar de la persona, pero estas iniciativas son aún minoritarias. Como indican Verdugo et al. (2009a) en su informe sobre el estado de las políticas de apoyo a la vida independiente de las personas con discapacidad, en España el énfasis se ha puesto claramente en los apoyos residenciales en vez de en aquellos basados en la comunidad y que fomentan la vida independiente y la inclusión social de las personas con discapacidad. Al margen de la fórmula de apoyo utilizada, los jóvenes con discapacidad experimentan numerosas barreras para emanciparse: casi dos tercios de los que tienen entre 27 y 30 años viven en el domicilio de sus padres (Huete et al., 2016).

En España, la vida independiente de las personas con discapacidad intelectual apenas ha sido objeto de investigación o estudio, aunque se han desarrollado distintas iniciativas a tener en cuenta. Así, en la línea de evaluación de la calidad de vida de las personas con DI atendidas en espacios residenciales destacan los trabajos desarrollados por investigadores del Instituto de Integración en la Comunidad (INICO) de la Universidad de Salamanca: entre ellos, el estudio de Gómez-Vela et al. (2002), orientado a evaluar la calidad de vida de adultos con DI usuarios de servicios residenciales en Castilla y León, y los instrumentos elaborados para evaluar la calidad de vida de adultos con discapacidad (Verdugo et al., 2009b, 2013 y 2014). Estos instrumentos permiten obtener datos relevantes a partir de los cuales emprender acciones que permitan mejorar el bienestar y calidad de vida de las personas con discapacidad intelectual. En el ámbito de la evaluación de los servicios, destacamos los trabajos de Navallas y Verdugo (2009), González Luna (2010) y Rojas (2004 y 2008), los tres centrados en contextos de viviendas y residencias para personas adultas con DI. En el caso de Navallas y Verdugo (2009), estos desarrollan un proyecto de innovación que tiene como objetivo mejorar la distribución de los apoyos en el ámbito de vivienda y residencia de una organización de Palma de Mallorca tomando como referencia el Modelo de Calidad de vida (Schalock y Verdugo). El estudio de González Luna (2010) se orienta a evaluar cómo el entorno de vivienda favorece la promoción de la autodeterminación de las personas, identificando facilitadores, barreras y buenas prácticas que orienten la innovación de la organización. Por su parte, Rojas (2004 y 2008) estudia los factores favorecedores de la autodeterminación y calidad de vida de personas que viven en un hogar tutelado en la comunidad, mostrando la relevancia que tienen las actitudes profesionales para garantizar la calidad de vida de los residentes.

1. Según datos del Instituto de Mayores y Servicios Sociales (IMSERSO 2013, en Huete y Quezada, 2014: 53), 1248 personas recibían en febrero de 2013 la prestación económica de asistencia personal, lo que representa sólo un 0,13\% del total de prestaciones del Sistema para la Autonomía y Atención a la Dependencia. El $88,5 \%$ de las personas que cuentan con esta prestación se encuentra en el País Vasco. 
En los últimos años, han incrementado los estudios que plantean alternativas y/u orientaciones para apoyar a las personas con DI a vivir en su propio hogar. En el ámbito de la formación para la vida independiente, destacan los trabajos de Illán y Molina (2013), centrado en un modelo formativo para la promoción de la vida autónoma e independiente; de Fernández Cordero (2013), que describe el proyecto ETXERATU, desarrollado a partir de tres programas que incluyen el aprendizaje para la vida independiente, el apoyo a la emancipación y apoyos a la autonomía en el hogar y entorno), y el de Vived et al. (2013), que describe ampliamente un programa de formación para la vida independiente, incluyendo los marcos referenciales, la propuesta curricular y el sistema de evaluación.

Se han realizado también estudios que evalúan la efectividad de experiencias vinculadas con los apoyos a la VI. Así, Liesa y Vived (2010) desarrollan un estudio de caso con el objeto de profundizar en los efectos que produce en jóvenes con DI su participación en un proyecto de vivienda compartida con estudiantes universitarios, concretando avances en el ámbito de las habilidades sociales y de la autonomía personal. Latorre y Liesa (2016), a partir de un estudio de caso centrado en participantes con DI en el mismo proyecto, indican avances en habilidades sociales, convivencia y ocio.

Las percepciones de las propias personas con discapacidad acerca de necesidades de apoyo y de sus familias son exploradas por Ruiz (2016), quien a partir de los resultados diseña y evalúa un modelo de asistencia personal que considera apropiado para favorecer la autonomía y la vida independiente. Por su parte, Puyaltó (2016) profundiza en las opiniones de personas con DI acerca de cuáles son las barreras con las que se encuentran para poder construir sus proyectos de vida y los apoyos que las ayudan. Entre las barreras, destacan la falta de oportunidades para tomar decisiones y las actitudes protectoras de familias y profesionales. Los participantes reclaman que estos les apoyen para avanzar en su autonomía y ampliar sus relaciones sociales.

La presente investigación tiene como objetivo analizar cuáles son, según las propias personas con discapacidad intelectual, sus familias y profesionales, las principales barreras a las que se enfrentan las personas con DI para construir sus proyectos de vida independiente, así como los apoyos que las ayudan a conseguirlos. Ello es clave para poder plantear propuestas de mejora que, a diferentes niveles (política, organización de servicios, acción socioeducativa, formación de profesionales, etc.) permitan avanzar en el cumplimiento del derecho a la vida independiente. 


\section{Método}

\subsection{Contexto de la investigación y objetivos}

En Cataluña, donde se desarrolla la investigación presentada², el Decreto 318/2006 de servicios de acogida de personas con discapacidad, establece las características de los dos modelos de atención que han sido mayoritarios hasta ahora: residencia y hogar de grupo. El hogar de grupo se caracteriza por ser un hogar en el que conviven un máximo de 12 personas de 18 a 65 años, con necesidad de apoyo intermitente, limitado, extenso o generalizado ${ }^{3}$. Además, existe desde el año 2002 el Programa de Apoyo a la Autonomía en el Propio Hogar (PAPH), destinado a un máximo de 4 personas de 18 a 65 años con necesidad de apoyo intermitente que viven en un hogar que es alquilado o propiedad de una de ellas. Este programa es regulado anualmente por la Generalitat de Cataluña, estableciendo tanto la cuantía de la ayuda como las condiciones que deben reunir los beneficiarios para poderla recibir (Ruf, 2006). La cuantía actual, 500 euros mensuales ${ }^{4}$ la solicitan directamente las personas con DI y, si es concedida, la recibe el servicio que va a prestar el apoyo (que debe estar previamente acreditado para poderlo ofrecer). En todos los casos, el acceso a estos servicios está condicionado por la valoración que hace la administración de la necesidad de apoyo de la persona, que no debe requerir más de 10 horas de apoyo a la semana.

Con el objeto de analizar la situación actual que viven las personas con DI en relación con la vida independiente, y plantear acciones orientadas a garantizar el logro del artículo 19 de la Convención, se llevó a cabo una investigación organizada en las siguientes fases:

1. Estudio cualitativo sobre los puntos de vista de las personas con DI.

2. Estudio cualitativo sobre las percepciones y puntos de vista de las familias.

3. Estudio cualitativo sobre las perspectivas de los profesionales expertos en los procesos de apoyo a las personas con DI.

\subsection{Participantes}

Participaron en la investigación 94 personas con discapacidad intelectual, 35 familiares y 33 profesionales. La tabla 1 muestra las principales características de los participantes en cada una de las fases del estudio.

2. Esta investigación ha sido parcialmente financiada por el MINECO (Gobierno Español) a través del proyecto EDU2014-55460-R. Tiene por título: "Los procesos de apoyo a la vida independiente de las personas con discapacidad intelectual en España: diagnóstico, buenas prácticas y diseño de un plan de mejora".

3. El Decreto 318/2006, en su artículo 3.2, establece los siguientes apoyos en función de su intensidad: el 'apoyo intermitente' que se presta de forma esporádica u episódica y que se proporciona cuando se necesita; el 'apoyo limitado' que se presta de forma ocasional y continuada durante un período de tiempo limitado, con una frecuencia regular, y en algunos de los entornos de la vida de la persona (hogar, trabajo, vida comunitaria, etc.); el 'apoyo extenso' que se presta de forma continuada, sin límite de tiempo, con una frecuencia regular o alta, y en algunos de los entornos de la vida de la persona, y el 'apoyo generalizado' que se presta de forma continuada, posiblemente durante toda la vida de la persona, con una frecuencia alta e intensa, y afecta a todas o casi todas las situaciones de vida de la persona.

4. Según establece el punto 13.2. de la Orden TSF/111/2017 de 7 de junio, por la cual se aprueban las bases para la concesión de las ayudas de apoyo a la autonomía en el propio hogar para el año 2017. 


\begin{tabular}{|c|c|c|c|}
\hline FASE & Objetivo & Participantes e instrumentos & Características participantes \\
\hline \multirow{2}{*}{1} & \multirow{2}{*}{$\begin{array}{l}\text { Conocer los puntos de vista de } \\
\text { las personas con DI sobre las } \\
\text { dificultades y apoyos hallados en el } \\
\text { desarrollo de proyectos hacia la vida } \\
\text { independiente. }\end{array}$} & $\begin{array}{l}22 \text { personas con DI participaron } \\
\text { en entrevistas individuales en } \\
\text { profundidad }\end{array}$ & $\begin{array}{l}\text { - } 11 \text { hombres y } 11 \text { mujeres con edades } \\
\text { entre } 21 \text { y } 55 \text { años. } \\
\text { - Distintas situaciones personales en } \\
\text { cuanto a vivienda (domicilio familiar, } \\
\text { propio, hogar tutelado, apoyo en el } \\
\text { propio hogar). }\end{array}$ \\
\hline & & $\begin{array}{l}72 \text { personas con DI participaron } \\
\text { en } 10 \text { grupos focales }\end{array}$ & $\begin{array}{l}\text { - } 38 \text { hombres y } 34 \text { mujeres miembros } \\
\text { de grupos de autogestión. } \\
\text { Distintas situaciones personales en } \\
\text { cuanto a vivienda (domicilio familiar, } \\
\text { propio, hogar tutelado, apoyo en el } \\
\text { propio hogar). }\end{array}$ \\
\hline 2 & $\begin{array}{l}\text { Conocer los puntos de vista de } \\
\text { las familias de personas con } \\
\text { DI acerca de las dificultades y } \\
\text { apoyos que encuentran sus hijos } \\
\text { e hijas (o familiares con DI) para } \\
\text { desarrollar proyectos hacia la vida } \\
\text { independiente }\end{array}$ & $\begin{array}{l}\mathbf{3 5} \text { familiares de personas con } \\
\text { DI (12 hombres y } 23 \text { mujeres) } \\
\text { participaron en } \mathbf{5} \text { grupos focales }\end{array}$ & $\begin{array}{l}\text { - } 12 \text { hombres y } 23 \text { mujeres, padres o } \\
\text { madres de personas con DI. }\end{array}$ \\
\hline 3 & $\begin{array}{l}\text { Conocer los puntos de vista los } \\
\text { profesionales que trabajan en } \\
\text { servicios de apoyo a las personas } \\
\text { con DI acerca de las dificultades } \\
\text { y apoyos que encuentran estas } \\
\text { personas para desarrollar proyectos } \\
\text { hacia la vida independiente }\end{array}$ & $\begin{array}{l}33 \text { profesionales vinculados } \\
\text { a la organización de servicios } \\
\text { relacionados con el apoyo } \\
\text { a personas adultas con DI } \\
\text { participaron en entrevistas } \\
\text { individuales semiestructuradas } \\
\text { en profundidad }\end{array}$ & $\begin{array}{l}\text { - } 25 \text { mujeres y } 8 \text { hombres con más de } \\
6 \text { años de experiencia en procesos } \\
\text { de apoyo a personas con DI. }\end{array}$ \\
\hline
\end{tabular}

Fuente: elaboración propia.

\subsection{Instrumentos y procedimiento}

La elección de entrevistas y de grupos focales para la recogida de información en esta investigación responde a la necesidad de conocer en profundidad los puntos de vista de personas con discapacidad intelectual, sus familias y los profesionales acerca del tema objeto de análisis. La entrevista individual y en profundidad permite a la persona entrevistada expresarse oralmente transmitiendo su definición personal de una situación al entrevistador, toma la forma de un relato narrado por la misma persona que lo ha experimentado. El entrevistador actúa como facilitador de la narración del entrevistado y ayuda a crear un clima adecuado para que este relato pueda tener lugar (Seidman, 2006). Las entrevistas en profundidad que se realizaron en este estudio tenían el objetivo de obtener los puntos de vista de profesionales y de personas con DI sobre distintos temas relacionados con los procesos hacia la vida independiente.

Las entrevistas empezaron a utilizarse en la investigación con personas con DI a mediados de los años 80 como método para conocer las vivencias de estas personas, habitualmente silenciadas en los enfoques metodológicos más tradicionales (Hollomotz, 2017). Beail y Williams (2014) destacan que es necesario tener en cuenta las habilidades lingüísticas de la persona que participa en la entrevista y poner especial cuidado 
en la forma en que se elaboran las preguntas y cómo estas pueden condicionar la participación de la persona con DI. Haya et al. (2014) sugieren que al realizar entrevistas con personas con DI hay que procurar ser flexibles a la hora de formular las preguntas (planteamiento de las preguntas de distintas formas, predominio de preguntas directas, coherencia con el modo de expresarse de la persona, etc.), recoger información sobre el lenguaje no verbal en la transcripción, utilizar los canales y apoyos comunicativos adecuados a cada persona, por ejemplo, el uso de imágenes por su potencial evocador, y sobre todo, procurar que la persona comprenda que tiene libertad para decidir qué preguntas desea contestar.

En cuanto a los grupos focales, Bloor et al. (2001) consideran que son un medio atractivo para la participación de personas en un proceso de investigación ya que representan acontecimientos sociales, limitados en el tiempo, que no requieren habilidades técnicas de las personas que participan. Los grupos de discusión ofrecen un entorno natural que permite que los participantes puedan hablar de sus percepciones, sentimientos y pensamientos sobre distintas cuestiones. Cambridge y McCarty (2001), Barr et al. (2010) y Kaehne y O'Connell (2010) subrayan el potencial de los grupos focales para favorecer la inclusión y el empoderamiento de las personas vulnerables y, en concreto, con personas con discapacidad intelectual. Plantean que los grupos focales constituyen un entorno no intimidatorio para la discusión que ayuda a los participantes a ganar confianza, facilitar y recibir apoyo por parte del resto de los participantes, y contrastar sus experiencias. Además, posibilita que ciertas personas con pocas habilidades de lectoescritura puedan participar activamente en procesos de investigación.

\section{- Fase 1}

La participación de las personas con DI en las investigaciones representa todavía un desafío en nuestro contexto, y algunos investigadores señalan la necesidad de desarrollar experiencias en que sus voces adquieran una mayor relevancia (González Luna, 2013; Haya et al., 2014; Susinos y Parrilla, 2008, 2013). Es en este sentido que en esta investigación, para conocer los puntos de vista de las personas con DI acerca de las dificultades y apoyos que encuentran en el desarrollo de sus proyectos de vida independiente, se utilizaron dos métodos, entrevistas individuales y grupos de discusión. En concreto, se llevaron a cabo 10 grupos focales y 22 entrevistas. Para el diseño de la entrevista se elaboró una lista de las temáticas más relevantes en relación a la vida independiente a partir de las aportaciones de la literatura. Una primera versión de la entrevista fue sometida a un doble proceso de validación. Por un lado, la entrevista fue revisada por 11 expertos en materia de inclusión social y discapacidad intelectual (4 profesionales de organizaciones proveedoras de servicios y 7 académicos). Por otro lado, se organizó una reunión con 5 asesores con DI que valoraron las preguntas de la entrevista mediante un grupo focal. En ambos casos se valoró tanto el contenido como la forma en la que se planteaban las preguntas. Tras incluir sus aportaciones, se elaboró el diseño final de la entrevista.

En el caso de las entrevistas, los participantes se seleccionaron mediante el contacto con distintas organizaciones del territorio proveedoras de servicios de apoyo a personas con DI. Las entrevistas se llevaron a cabo de febrero a septiembre de 2015. Se desarrollaron individualmente, en los espacios donde la persona entrevistada decidió ser entrevistada. De este modo se llevaron a cabo 10 encuentros en la propia sede de la organización, 8 en la Universidad y 4 en el propio hogar de la persona. La duración media de las entrevistas fue de hora y cuarto. Se abordaron los siguientes temas:

- La vivienda: situación actual y deseada, la toma de decisiones sobre la vivienda, barreras encontradas y apoyos recibidos y deseados en relación con la vivienda. 
- La participación en la comunidad: participación actual y deseada, la toma de decisiones en cuanto a la participación en la comunidad, barreras encontradas y apoyos actuales y deseados.

- Las relaciones afectivas: situación actual y deseada, barreras identificadas y apoyos actuales y deseados para iniciar y mantener relaciones afectivas.

- Acciones que pueden contribuir a que las personas con DI vivan dónde quieran, cómo quieran y con quién quieran.

En los grupos focales participaron 72 personas miembros de distintos grupos de autogestión del territorio donde se llevó a cabo la investigación. A través de la federación territorial de organizaciones dirigidas a las personas con $\mathrm{DI}$, se contactó con los distintos grupos de autogestión de la zona. Fueron 10 los grupos que accedieron a participar en la investigación. Todos los grupos tenían más de un año de funcionamiento y estaban vinculados a alguna organización de atención a personas adultas con discapacidad. El objetivo del grupo focal era aportar reflexiones y opiniones sobre el papel de los profesionales en el apoyo a las personas con discapacidad en los procesos de emancipación y vida independiente a partir de las experiencias propias y de compañeros. Los grupos focales se realizaron entre noviembre de 2015 y junio de 2016. Se desarrollaron en el lugar donde se reúnen habitualmente los autogestores (generalmente espacios de las entidades o centros cívicos) y tuvieron una duración media de dos horas. Se trataron los siguientes temas:

- Las opciones actuales de apoyo a la vida independiente.

- El papel de los profesionales, de las familias y de las propias personas con DI en la determinación y elección del proyecto de vida de la persona y de los apoyos necesarios para lograrlo.

- La actuación de las organizaciones proveedoras de servicios en la transición hacia un modelo de apoyo basado en la comunidad.

- Las redes de apoyo informales.

Previamente a la realización de los grupos focales, un grupo de asesores con discapacidad llevó a cabo, mediante un grupo focal, la validación de los temas y del documento-guía que fue utilizado en el grupo de discusión.

\section{- $\quad$ Fase 2}

El objetivo de la fase 2 era conocer los puntos de vista de las familias de personas con DI acerca de las dificultades y apoyos que encuentran sus hijos para desarrollar proyectos de vida independiente. A través de organizaciones que ofrecen servicios a personas adultas se invitó a las familias a participar. Accedieron a ello 35 personas, 12 hombres y 23 mujeres, que eran madres o padres de personas con DI. Se distribuyeron en 5 grupos focales, los cuales se llevaron a cabo entre los meses de abril a junio del 2016 tratando los mismos temas que en los grupos focales anteriores. Todos los grupos focales se llevaron a cabo en espacios de entidades, y tuvieron una duración media de dos horas.

\section{- Fase 3}

Con el objeto de conocer los puntos de vista de los profesionales que trabajan en servicios de apoyo a las personas con $\mathrm{Dl}$ acerca de las dificultades y apoyos que encuentran estas personas para desarrollar pro- 
yectos hacia la vida independiente se recurrió también a entrevistas semiestructuradas en profundidad. Se diseñó la entrevista a partir de los siguientes temas: valoración de las opciones actuales de apoyo a la VI, el papel de los profesionales, el papel de las organizaciones, el papel de las familias, el papel de las propias personas con DI, el papel de la red de apoyo informal (amigos). Se realizó un pilotaje consistente en la aplicación de las entrevistas a tres profesionales, tras el cual se diseñó el instrumento definitivo. Las entrevistas se llevaron a cabo desde noviembre del 2015 a julio del 2016, en los servicios donde desempeñan su trabajo los profesionales y tuvieron una duración media de una hora y cuarto.

\subsection{Análisis de datos}

Todos los participantes fueron informados de los objetivos de la investigación y firmaron un documento de consentimiento informado que les solicitaba permiso para grabar sus narraciones, y les garantizaba el anonimato y la recepción de información acerca de los resultados de la investigación. Las entrevistas y grupos focales fueron transcritos y posteriormente analizados mediante la técnica del análisis de contenido temático, método de análisis cualitativo que permite tomar en consideración los temas relevantes de un conjunto de datos, de acuerdo con el tema de investigación (Guest et al., 2012), combinando un proceso de codificación estructural con un proceso descriptivo (Saldaña, 2013). Se partió de una lista provisional de códigos basada en los temas tratados en las entrevistas y grupos focales, que se amplió con códigos descriptivos surgidos de la revisión continua de las transcripciones. Seguidamente se analizaron independientemente los datos de cada grupo (personas con DI, familias y profesionales) codificando los fragmentos de acuerdo con este listado. La tabla 2 muestra los temas y subtemas a partir de los cuales se organizaron los códigos.

\begin{tabular}{|c|c|}
\hline Temas & Subtemas \\
\hline Opciones/alternativas de vida & $\begin{array}{l}\text { - Fortalezas y debilidades de las distintas opciones (hogar de grupo, apoyo en el propio } \\
\text { hogar) para potenciar la VI, la participación e inclusión social } \\
\text { - Barreras para acceder a los apoyos que potencien el estilo de vida deseado } \\
\text { (participación, relaciones afectivas, vivienda) }\end{array}$ \\
\hline Rol de los profesionales & $\begin{array}{l}\text { - Acciones de apoyo efectivo para la VI } \\
\text { - Habilidades profesionales más valoradas } \\
\text { - Propuestas para la transformación de los apoyos }\end{array}$ \\
\hline $\begin{array}{l}\text { Rol de las organizaciones de } \\
\text { apoyo }\end{array}$ & $\begin{array}{l}\text { - Propuestas para la transformación de las organizaciones } \\
\text { - Relación profesional-organización }\end{array}$ \\
\hline Papel de la familia & $\begin{array}{l}\text { - Acciones de apoyo efectivo } \\
\text { - Relación profesional-familia. Trabajo con las familias } \\
\text { - Propuestas de mejora }\end{array}$ \\
\hline Papel de la persona & $\begin{array}{l}\text { - Concreción del papel actual de la persona } \\
\text { - Propuestas de cambio/mejora } \\
\text { - Necesidades de apoyo para desarrollar un papel más activo }\end{array}$ \\
\hline $\begin{array}{l}\text { Papel de la red de apoyo } \\
\text { informal }\end{array}$ & $\begin{array}{l}\text { - Papel de las amistades } \\
\text { - Dificultades para construir redes de apoyo } \\
\text { - Apoyos necesarios para construir y mantener redes de apoyo }\end{array}$ \\
\hline
\end{tabular}

Fuente: elaboración propia. 
En este artículo se van a abordar concretamente los siguientes ejes temáticos vinculados con el objeto de estudio:

- Formación y preparación para la vida independiente.

- La relación con los profesionales.

- Los ingresos económicos.

- El modelo de organización de los apoyos.

- El papel de la familia.

Disponer de distintas fuentes de datos, utilizar distintos métodos, varios marcos teóricos y diversos investigadores, se considera valioso en el campo de la investigación cualitativa porque permite captar distintas dimensiones de los problemas, aumenta el alcance de la investigación y ayuda a profundizar en la comprensión del problema. Es lo que se denomina triangulación y se considera que favorece la credibilidad de la investigación, uno de los criterios de calidad en la investigación cualitativa (Tracy, 2010). En la investigación que se presenta, la información procede de distintas fuentes (personas con DI, familiares y profesionales), se obtiene con distintos instrumentos (entrevistas en profundidad y grupos focales) y es analizada por varios investigadores, combinando el trabajo individual de análisis con el trabajo en equipo y llegando finalmente a un consenso. La triangulación de la información ha permitido identificar las cuestiones y preocupaciones prioritarias de los distintos temas. Además, la obtención de información de personas con discapacidad, familiares y profesionales ha facilitado lo que Tracy (2010) denomina multivocality, es decir, la posibilidad de ofrecer una visión del tema desde las voces de los distintos implicados.

\section{Barreras y apoyos para la vida independiente: opiniones de personas con discapacidad intelectual, familias y profesionales}

Seguidamente se presentan los principales resultados para los distintos temas planteados. Para cada uno de ellos se indican las principales barreras y se aportan citas extraídas de las entrevistas y grupos focales con las personas con $\mathrm{DI}$, de las entrevistas a los profesionales y de los grupos focales con las familias.

\section{- Formación y preparación para la vida independiente}

La orientación e información previa tanto a usuarios como a sus familias constituye una de las acciones bien valoradas tanto por parte de profesionales como por las propias personas con discapacidad y sus familias. Los profesionales aludieron a la necesidad de trabajar con las familias para vencer actitudes de sobreprotección y los miedos de las familias y las propias personas. Las familias valoraron muy positivamente la pedagogía impulsada desde las instituciones para apoyar su propia disposición en relación con los procesos de vida independiente de sus hijos e hijas. Los autogestores apreciaron la información facilitada por los profesionales para valorar, conjuntamente con sus familias, las posibilidades de emancipación. El apoyo de los profesionales en este sentido es importante, puesto que una de las principales barreras para 
la vida independiente según las personas con DI es no sentirse lo suficientemente preparadas a la hora de emprender sus proyectos de vida:

"Más de una vez me lo he planteado (emanciparme de casa de mis padres), pero quieras o no, sí que me lo planteo, pero me hace como cosa. O sea, me da miedo. No sé realmente como irá, si yo estaré viviendo solo o con un compañero o quién sea...".

“¿Qué es lo que te preocupa más? ¿Qué es lo que crees que te puede resultar más difícil viviendo solo?”.

"Pues vivir solo, hacer las cosas por mí mismo, que todavía veo que no sé mucho".

(Pau, 25 años, entrevista individual).

En este sentido, tanto las personas con discapacidad como los profesionales valoran especialmente que la formación pueda ayudarles a ganar confianza y autonomía. Las personas que reciben apoyo en el propio hogar valoran especialmente el apoyo que reciben para "el papeleo" y la gestión de la economía doméstica. Reconocer la importancia de la formación no la exime de críticas, ejercidas básicamente por los profesionales y las familias. Los primeros plantean que las acciones formativas se diseñan a menudo a partir de las demandas puntuales de las personas sin obedecer a una planificación o proyecto específicamente orientado a la preparación para la vida independiente:

"No tenemos un plan de formación para ellos (...), se trabajan temas de formación, temas que siempre se abordan y que además piden ellos, son temas de sexualidad, temas de higiene, habilidades sociales, se trabajan temas de comunicación para los que tienen dificultades... pero de forma informal, bien organizado no lo tenemos hecho...". (Profesional 2).

Además, el hecho de que parte de las acciones formativas que se realizan tengan lugar de forma previa en el contexto de la propia organización, dificulta su aplicabilidad en el ámbito comunitario:

"Yo creo que para la vida independiente no se les prepara, ni a la escuela ni nosotros tampoco realmente... Hacemos talleres de habilidades domésticas, de cocina... pero claro, se quedan dentro del servicio".

(Profesional 1).

Por su parte, las familias reivindican la necesidad de que los procesos de formación para la vida independiente se planteen no solo en contextos reales, sino que se articulen de manera que las personas con DI puedan vivir y experimentar durante un periodo relativamente extenso de tiempo lo que suponen estos procesos. Plantear la formación para la vida independiente en contextos limitados a la propia organización puede resultar contraproducente porque no favorece el empoderamiento en relación con las propias potencialidades ni facilita la toma de conciencia de la necesidad de vivir una vida propia e independiente. Las familias valoran que vivir en una vivienda compartida, de forma temporal, ayuda a tomar consciencia de las propias posibilidades, necesidades e intereses, lejos de la sobreprotección de las familias. Por ejemplo:

“...en el piso saben que les toca hacer la comida porque es su responsabilidad...".

(Familia, GF2).

Por último, en los grupos de discusión con familias también aparece de forma recurrente la importancia de potenciar los procesos de vida independiente desde las etapas de escolarización obligatoria. 


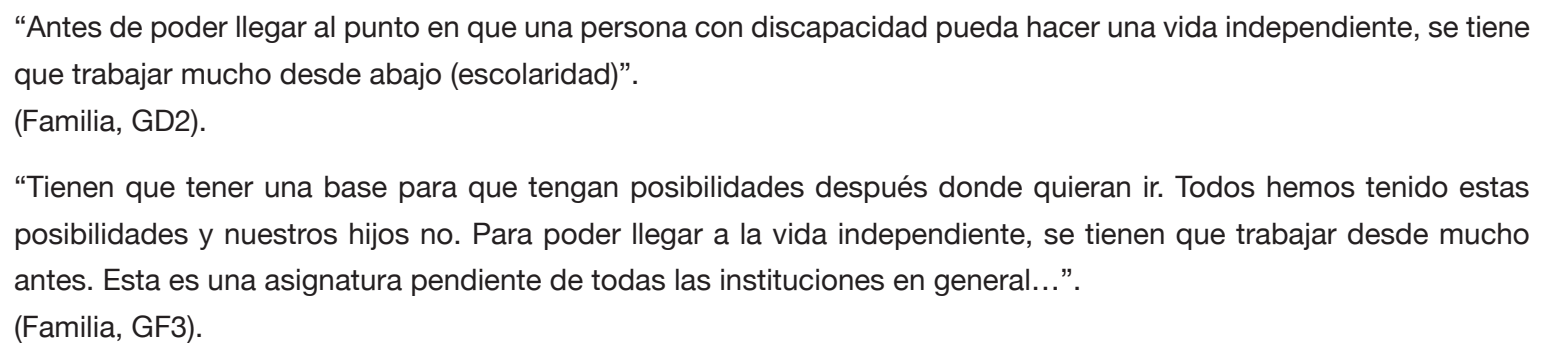

\section{- La relación con los profesionales}

Los profesionales juegan un papel fundamental en el apoyo a los procesos de vida independiente, y en ello coinciden tanto personas con DI como familias. Las personas con DI valoran en general de forma positiva el apoyo emocional recibido por parte de los profesionales; se valora que el profesional ejerza este acompañamiento emocional, con lo que acaba convirtiéndose en una figura próxima a la que comparan con frecuencia con un familiar o amigo. En este sentido, valoran especialmente las habilidades interpersonales relacionadas con la escucha y la empatía, es decir, que los profesionales tengan paciencia, que les inspiren confianza y les ofrezcan un apoyo incondicional:

"que tengan mucha paciencia...".

"Y que sea tranquilo".

"Que te escuchen, te ayuden, y comprendan tus problemas".

"Y confianza... cuando llevas muchos años con ellos ya parece que son de la familia".

(Autogestores, GF3).

Por su parte, las familias valoran especialmente a los profesionales, sobre todo cuando en la entidad existe una estructura organizativa que facilita el intercambio de información entre la familia y los profesionales.

"Te dicen lo bueno y lo malo... te dicen que estrategia tienes que seguir... te dicen que si sabes alguna cosa se lo comuniques. Veo detalles que me indican que realmente les preocupa mi hijo. Y esto me deja más tranquila...”. (Familia, GF1).

"Es que estos apoyos los han de transmitir a las personas que están alrededor de la persona con DI, porque no sirve de nada que en la entidad se esté dando una formación concreta y que lleguen a casa y que esta formación desaparezca".

(Familia GF2).

Las intervenciones de las familias en los grupos de discusión nos indican que las variables que más se valoran de los profesionales son el conocimiento de las personas y de sus procesos vitales y el respeto hacia ellos. De la misma forma se refieren a la necesidad de la exigencia para fomentar los procesos de autogestión a través del razonamiento y la reflexión.

"Son personas que son capaces de hacer razonar y llegar a unas reflexiones con nuestros hijos. Esta es una actitud muy difícil de mantener y que lo consiguen... Tienen esta capacidad de decir que esto no es correcto del todo y ellos no se frustran. Este es el punto fuerte de los profesionales y técnicos".

(Familia, GF2). 
En cuanto a los ámbitos de apoyo, se considera positivo que el profesional, en función de las demandas de la propia persona, movilice los apoyos necesarios, ya sea para gestionar su propio dinero o para aumentar sus relaciones sociales. Así mismo, las personas con DI valoran positivamente que los profesionales de apoyo garanticen y faciliten el acceso a los distintos servicios que promueven la salud de la persona.

A pesar de haber avanzado en los planteamientos del modelo social, aún se mantienen estilos de actuación profesional poco centrados en la persona, que pueden dificultar el logro de los proyectos vitales de las personas con DI. En ello coinciden tanto profesionales como personas con discapacidad y familias. Los profesionales opinan que la cultura del servicio, en algunos casos en los que predomina el modelo institucional, puede condicionar sus actuaciones profesionales. También se mencionan los propios estereotipos y prejuicios:

\footnotetext{
"Nuestra sociedad tiene prejuicios, estigmas, estereotipos, y nosotros los tenemos incorporados y no somos conscientes... en el fondo los profesionales y las familias somos conservadores, somos proteccionistas, en el fondo creemos poco en las posibilidades de las personas...".

(Profesional 7).
}

Incluso en el caso de los profesionales que ofrecen apoyo en el propio hogar, éstos consideran que, para llevar a cabo un apoyo personalizado centrado en la persona que vaya más allá del apoyo a las necesidades básicas de la vida diaria y que se ocupe de formar en autogestión y en la toma de decisiones propias, es necesario disponer de mayores recursos profesionales.

\footnotetext{
“...este tipo de acompañamiento es muy limitado, porque sólo son unas horas limitadas, y seguramente para que la persona pueda llevar una vida independiente es necesario trabajar en otras áreas de su vida que, a lo mejor, desde el apoyo no se te permite porque no dispones de suficientes horas, desde trabajar la autogestión, el derecho a decidir (...). Tú ves a la persona y dices: ¿qué le falta a esta persona? Apoyo para ir a comprar, apoyo para cocinar... y priorizas estos hábitos del día a día, pero hay una segunda parte de la persona que debería trabajarse y que no se está trabajando".

(Profesional 3).
}

En cuanto a las críticas, los autogestores han manifestado en algunos de los grupos de discusión que a menudo sienten que no tienen apoyo suficiente y lo relacionan también con la falta de personal.

"A mí me gustaría mucho que hubiera dos personas de apoyo en el hogar. Para que cuando una estuviera de vacaciones, pudiese venir la otra. O pudieran turnarse. Por ejemplo, la semana que viene, mi persona de apoyo en el hogar tiene vacaciones y no podrá acompañarme al psiquiatra, y me hubiera gustado, porque ahora se trata de un nuevo tratamiento, y a ver qué será".

(Autogestores, GF6).

En el mismo sentido que los profesionales y que los autogestores, algunas familias se refieren a que se tiende a homogeneizar a todas las personas del grupo como consecuencia de la falta de recursos (económicos, humanos):

"A los profesionales les cuesta adaptarse a las diferencias de todos los chicos porque si tienen una ratio de 8 pues hacen un sistema que más o menos les pueda ir bien a todos. Y claro, esto es muy poco flexible".

(Familia, GF5) 


\section{- Los ingresos económicos}

Independientemente del ámbito laboral en el que trabaja la persona con DI (ordinario o protegido), los ingresos económicos representan una barrera importante a la hora de plantear posibles proyectos de vida. Este es un tema que plantean abiertamente tanto las personas con DI, como los profesionales y las familias participantes en la investigación. El sueldo de las personas con DI que trabajan en centros especiales de empleo es equivalente al salario mínimo interprofesional y en el caso de las personas que trabajan en empresas ordinarias, la mayoría de ellas trabajan a tiempo parcial, siendo éste el motivo por el que algunas de las personas participantes han desistido de sus proyectos de emancipación.

\footnotetext{
"Nos encontramos que para muchas personas dar un paso adelante en el tema de vida independiente no es posible por la capacidad económica. Porque, aunque estén trabajando, la vivienda es cara y están muy lejos de esta posibilidad".

(Profesional 8).
}

Las familias se refieren reiteradamente a que la posibilidad de vida independiente está condicionada a un trabajo. En caso contrario, la familia tiene que asumir todos los gastos y esto es, en la mayoría de los casos, inviable. En todos los grupos de discusión se reivindica la necesidad de que las opciones de vida independiente sean sostenibles para las personas con DI. Por tanto, se establece una relación directa entre procesos de vida independiente y acceso a un trabajo suficientemente remunerado para mantener esta opción.

\footnotetext{
"Sí, el trabajo (imprescindible). Es lo que hay porque no se pueden permitir ellos todos los gastos. Porque ahora sí que los podemos ayudar, pero ¿y cuando no podamos ayudarlos que pasará? Si no cobran el dinero para poder mantenerse ellas solas pues no podrán estar. Y las ganas las tienen, pero lo malo es que no lleguen”.

(Familia, GF2).
}

Los escasos ingresos económicos condicionan que, en ocasiones, no se puedan llevar a cabo los proyectos de emancipación que conducen a vivir solo o en pareja, sino que éstos se traduzcan en compartir la vivienda con otras personas que se hallan en la misma situación (en el PAPH las unidades de convivencia pueden ser de hasta cuatro personas, todas beneficiarias del mismo programa).

Además, los bajos ingresos económicos de las personas con DI no sólo condicionan sus procesos de emancipación y el hecho de decidir dónde y con quién vivir, sino que además repercuten en sus posibilidades para acceder a formación y participar en actividades de la comunidad.

"A mí me hubiera gustado (estudiar) educación infantil. (...) Pero claro, como no ha podido ser, he mirado de sacarme el diploma de monitora, pero como es muy caro, no puedo hacerlo". (Íngrid, 26 años, entrevista individual).

Para superar esta barrera, las personas con DI consideran que su situación mejoraría si los precios en general y especialmente de las viviendas fueran más asequibles. También se reclama el acceso a vivienda social. 


\section{- El modelo de organización de los apoyos}

De los hogares de grupo, las personas denuncian la rigidez de sus normativas, la falta de intimidad, la convivencia con muchas personas y la falta de atención individualizada. Por su parte, en algunas de las entrevistas, los profesionales aluden a la necesidad de respetar la privacidad de los residentes en los hogares de grupo, aunque es difícil compatibilizar este derecho con el funcionamiento del hogar:

\footnotetext{
"Sería ideal tener hogares de grupo de 6 a 8 personas, todos con habitación individual, que se respetara al máximo la privacidad, pero la realidad no es esta, tú tienes que hacer sostenible el servicio y debes tener una ratio funcional ${ }^{5} . .$. . (Profesional 4).

"En los hogares de grupo hay un mínimo de normas internas de funcionamiento, como ir a dormir más o menos a la misma hora... Claro, hay unos mínimos que se deben cumplir".

(Profesional 5).
}

Muchos de los profesionales entrevistados valoran que el modelo de apoyo a la vivienda ofrecido por las organizaciones que trabajan con las personas con DI deriva claramente del modelo individual o médico y es, hoy en día, excesivamente rígido.

"Creo que se deberían poder introducir cambios en este modelo tan rígido que llevarían a que, a la larga, una persona que quizás está viviendo en un hogar de grupo, pueda plantear: pues quiero ir a vivir sola, o en pareja... porque en la actualidad lo que pasa es que, como la persona ya está en el servicio, pues allí se queda, no avanza, ha conseguido salir de casa y parece que ya es independiente, aunque hay muchas otras opciones...".

(Profesional 8).

El Programa de Apoyo a la Autonomía en el Propio Hogar (PAPH), basado en apoyos personalizados, es valorado positivamente por parte de todos los profesionales entrevistados por tener una orientación claramente comunitaria; por su coste, más económico que el hogar de grupo, y también por el hecho de que sea la persona quien deba solicitar la ayuda, puesto que ello la sitúa en una posición de control del apoyo que recibe:

"Este programa ideológicamente es muy potente, porque quien realiza la demanda del recurso es la persona con discapacidad, y quien puede dejarlo cuando quiere también es la propia persona".

(Profesional 11).

Sin embargo, la normativa de este programa genera bastantes críticas. Una de ellas, el hecho de que las viviendas compartidas por cuatro personas - una de las agrupaciones posibles bajo este programa- no responden a la filosofía de vida independiente. Más que una vivienda independiente, lo que se establece es una mini-residencia.

"Estas unidades de convivencia de cuatro personas no son exactamente un servicio residencial, porque la estructura es diferente, pero la vida que hay dentro... es decir, cuando es la entidad quien alquila el piso, quien ofrece las plazas y quien sustituye las que quedan vacantes... esto es un hogar de grupo".

(Profesional 12).

5. Según el Decreto 318/2006 de 25 de julio, puede haber hasta 12 personas en los hogares de grupo, con un máximo del $20 \%$ de las habitaciones para uso individual. 
También se critica que los criterios de acceso son muy restrictivos: sólo pueden acceder a la ayuda las personas que, entre otros criterios, puedan necesitar un máximo de 10 horas de apoyo semanales. Ello deja fuera del programa a muchas personas que necesitarían, al menos al principio, más horas de apoyo.

"El programa como filosofía está muy bien, pienso que aporta muchas cosas buenas y otorga más autonomía a la propia persona, pero llega un momento que tantos criterios, tantos requisitos, temporalización... el programa está muy acotado, no hay flexibilización, el tope es 10 horas de apoyo por persona, ¿y el que necesita 15 horas? ¿o el que necesita menos?".

(Profesional 13).

"Se debería poder ofrecer el apoyo evidentemente a cualquier persona independientemente del nivel de apoyo, de la intensidad de nivel de apoyo que necesite...".

(Profesional 14).

El programa se convoca anualmente, desde su creación en el año 2002, y la partida presupuestaria que le corresponde varía en función del presupuesto autonómico. En los últimos años los criterios de acceso se han restringido y también el importe que se adjudica a la persona para el apoyo. Los profesionales reclaman que el programa pase a ser un servicio social que no dependa de la disponibilidad presupuestaria anual.

“El programa surgió el 2002 y aún no ha pasado a ser un servicio... depende anualmente de que la Generalitat saque una partida presupuestaria para este programa. No hay el mismo dinero cada año, depende de lo que la Generalitat vaya sacando... hace muchos años que los profesionales estamos reclamando que pase a ser un servicio".

(Profesional 11).

Las familias, por su parte, explicitan que las opciones existentes ni son suficientes ni ofrecen una respuesta adecuada a la variedad de especificidades que presentan las personas con DI. Como consecuencia de esta falta de diversificación, las familias hacen referencia a que se encuentran en la tesitura de cubrir las carencias de los servicios.

“... hay pocos recursos y además hay lista de espera... el año que salió C. (su hija) había como muchas plazas. Entonces sacaron a todos los niños que cumplían años, algunos de ellos inmaduros, como el caso de C. Y entonces dijeron que más valía que entraran ahora, porque no nos podían asegurar que dentro de dos años hubiera plaza, porque si no nos la tendríamos que quedar en casa".

(Familia, GF3).

Otra de las dificultades que se entrevén en los grupos focales de familias se relaciona con la dificultad de acceder a la información existente. Este hecho aumenta la sensación de vulnerabilidad tanto de las familias como de las personas con DI:

"Para mí siempre era muy difícil encontrar información. Los familiares a veces me explican más cosas de las que yo podía encontrar en los servicios sociales... Hay mucha falta de información".

(Familia, GF5).

Para superar estas barreras, las familias apuntan la necesidad de que los servicios se coordinen entre ellos.

"Insisto, hace falta educación, concienciación y sensibilización en centros especiales y ordinarios para que todos estos centros funcionen integrados, por sí solos ellos ya funcionan, pero viven aislados...”.

(Familia, GF3). 


\section{- El papel de la familia}

El rol de la familia como fuente de apoyo en la vida independiente de la persona con DI es indiscutible, y así lo reconocen tanto los profesionales como las propias personas con discapacidad. Los profesionales coinciden en manifestar que cada vez hay mayor sensibilización en las familias sobre la posibilidad de que las personas con DI vivan fuera del hogar familiar, aunque ello no quiere decir que se plantee siempre como una necesidad vinculada a un proyecto de vida en el que la persona con DI sea la protagonista. Existe una coincidencia entre los profesionales en valorar que la familia suele preferir un modelo de vivienda compartida y, en muchos casos, el hogar de grupo, por la seguridad que ofrece el hecho de vivir en compañía de otras personas y con la presencia constante de profesionales de apoyo.

Los autogestores coinciden en subrayar el importante papel que juega la familia en sus vidas, sobre todo en cuanto a fuente de preparación para cuando se emancipen. Visualizan el hogar familiar como espacio donde se pueden dar las oportunidades para practicar y aprender aquellas habilidades vinculadas con la autonomía en el hogar: cocinar, limpiar y ordenar las habitaciones, etc. También las familias son conscientes del importante rol que juegan como espacio de potenciación de la autonomía personal de sus familiares con DI:

\footnotetext{
"Trabajar los hábitos, metodologías... que conozcan diferentes formas de hacer las cosas (de casa) y que tengan los apoyos que ellos puedan utilizar. Y el apoyo de la familia".

(Familia, GF3).
}

En algunos casos, también se identificó el hogar familiar como zona de confort, confianza y seguridad con la que se cuenta para plantear e iniciar proyectos de vida. En este sentido, en algunos grupos focales realizados con familias se explicita que es importante tener en cuenta que los procesos de vida independiente implican más que el hecho de dejar atrás el hábitat familiar. Supone respetar, por parte de las familias, decisiones, puntos de vista, intereses del familiar con DI. Sin embargo, tanto profesionales como autogestores coinciden en valorar que existe todavía una sobreprotección evidente por parte de la mayoría de las familias. En este sentido, una reclamación formulada en la mayoría de los grupos de discusión es que los padres (y otros familiares) no ejerzan esta sobreprotección que les impide tomar las propias decisiones en distintos ámbitos vitales:

\footnotetext{
"Nos ayudarían más (las familias) si no los tuviéramos siempre tan encima, que ya somos mayores...". (Autogestor, GF4).

A pesar de los avances en el apoyo familiar, aún en muchos casos los planes de futuro sobre la emancipación están condicionados por las circunstancias familiares y las decisiones tomadas por parte de las familias:

"Mi madre murió, vivo con mi padre, y si mi padre muere, que no sé cuando, pues viviré con mi hermana y mi cuñado".

(Autogestor, GF8).
}

Se ha puesto de manifiesto que las necesidades de las familias cambian con los años y el tema de la emancipación debe abordarse desde un planteamiento integral de orientación y apoyo, contando con las familias y sobre todo aportando recursos para que éstas puedan ayudar a sus hijos o familiares con discapacidad a establecer su propio proyecto de vida. 


\section{Discusión: conclusiones y propuestas}

Los datos aportados por la investigación son especialmente importantes en momentos como el actual, en el que existe una clara voluntad de avanzar en la inclusión social de las personas con DI de acuerdo con la Convención sobre los Derechos de las Personas con Discapacidad. En este sentido, los resultados de esta investigación ayudan a valorar cuáles son no sólo los retos sino también las acciones a plantear para que los proyectos de vida independiente de las personas con DI se puedan conseguir en coherencia con los planteamientos del art. 19 de la CDPD. A continuación, se sintetizan en cuatro puntos los posibles retos y acciones para avanzar hacia el ejercicio pleno de este derecho.

Una primera observación se refiere a la necesidad de incorporar la vida independiente como un derecho, y no como un privilegio al que pueden acceder aquellas personas que tienen menos necesidad de apoyos. Como plantean McConkey et al. (2016), el grado o nivel de apoyo necesario para las personas con DI determina con frecuencia las decisiones sobre las opciones de apoyo en el ámbito residencial. En nuestra investigación se confirma que éste es también el criterio dominante. Aunque ello obedece a una lógica economicista, no responde a un sistema organizado según los derechos de las personas, puesto que restringe el acceso a la vida independiente a aquellas que necesitan menos apoyo en su vida diaria y que disponen de los recursos económicos suficientes para acceder a una vivienda. En consecuencia, el sistema de provisión de servicios para la vida independiente debería reorientarse acentuando el acceso a los apoyos personalizados y a la vivienda a todas las personas con discapacidad que lo deseen. Diversas investigaciones y experiencias (Klein, 2000; Tøssebro et al., 2012; McConkey et al., 2016) muestran que, con los apoyos personalizados adecuados, personas con DI que por la valoración de su grado de autonomía serían asignadas a residencias u hogares de grupo, pueden pasar a vivir en su propio hogar.

Como en el estudio de García Iriarte et al. (2016), los propios profesionales se muestran conscientes de la necesidad de organizar apoyos personalizados y reconocen, al igual que en el estudio de McConkey y Collins (2010), que en ocasiones es la propia institución la que constituye un obstáculo para transformar el modelo de atención y abandonar la visión asistencialista que ha impregnado durante décadas la cultura de los servicios. Ello tiene implicaciones en las propuestas formativas que se realizarán seguidamente.

En segundo lugar, los profesionales constituyen un agente fundamental para garantizar los apoyos que hagan efectiva la vida independiente y la inclusión en la comunidad de las personas con DI. Profesionales, personas con discapacidad y familias coinciden en valorar positivamente el apoyo en la realización de actividades domésticas, pero apenas se menciona la necesidad de implementar acciones de apoyo dirigidas a potenciar la red social y la inclusión social en la comunidad. Ello coincide con otros estudios que estudian las valoraciones de profesionales (Clement y Bigby, 2012; McConkey y Collins 2010). Sin embargo, la vida independiente no supone únicamente desarrollarse autónomamente en una vivienda individual, sino que plantea otros retos como establecer relaciones personales y sociales significativas, participar en los servicios culturales y de ocio de la comunidad, entre otros. De acuerdo con los planteamientos de Ruf (2006), las relaciones sociales juegan un papel fundamental como apoyos a la autonomía personal en contextos de inclusión social, lo que argumenta el hecho de que se generan nuevos roles profesionales destinados a potenciar los vínculos sociopersonales con la comunidad (Duggan y Linehan, 2013; García Iriarte et al., 2016). Con ello se abre una gama de posibilidades de apoyo por parte de los profesionales, que hasta 
hace poco apenas se consideraban puesto que su trabajo se desarrollaba fundamentalmente en el contexto de los centros socioeducativos.

Las personas con discapacidad participantes en el estudio valoraron especialmente el apoyo emocional que reciben de sus profesionales de apoyo, resultado que coincide con otros estudios centrados en percepciones de usuarios con discapacidad (Clarkson et al., 2009; Roeden et al., 2011). En este sentido, ciertas competencias interpersonales como la empatía, la escucha, el respeto, la accesibilidad, etc., cobran una relevancia significativa y se configuran como habilidades a potenciar desde los procesos formativos (como también se plantea en Dodevska y Vassos, 2013) y a valorar en el reclutamiento de profesionales de apoyo. Asimismo, el papel de las familias en los procesos de emancipación requiere dotar a los profesionales de las competencias relacionadas con el trabajo con las familias para que éstas puedan constituir fuentes de apoyo (Bowey et al. 2005).

En tercer lugar, la necesidad de un compromiso firme por parte de la Administración que garantice la continuidad y estabilidad de los apoyos personalizados. Desde el inicio de la crisis económica que sacude a los países occidentales en los últimos años, los gobiernos han desarrollado políticas de reducción del gasto, siendo los servicios sociales uno de los ámbitos que mayores recortes han presenciado (European Commission, 2009). Estos recortes no sólo han perjudicado las partidas presupuestarias destinadas a los servicios sociales sino también los sueldos y la estabilidad laboral de los profesionales que, a través de estos servicios, brindan apoyo a las personas con DI. Ello ha ocurrido en nuestro contexto y también es señalado como un hecho constatable en otros países como Reino Unido (Hatton et al., 2009; Hawkins et al., 2011). La precariedad de los profesionales afecta a la calidad de los apoyos ofrecidos, especialmente de los apoyos personalizados y es un punto en el que incidir necesariamente para mejorar las posibilidades de vida independiente de las personas con discapacidad.

Por último, la persona no puede ejercer el derecho a la vida independiente si no puede gozar del resto de sus derechos. El derecho a la vida independiente va más allá de la desinstitucionalización y las opciones de apoyo, a pesar de que estos elementos sean indispensables (European Union Agency for Fundamental Rights, 2012). El derecho a tomar las propias decisiones, a tener una familia propia o tener un trabajo ajustado a las propias necesidades son otros de los muchos elementos que contribuyen a que una persona pueda vivir de forma independiente. Para avanzar en el ejercicio del derecho a la vida independiente es necesario abordar la gestión del riesgo desde perspectivas y estrategias que no impliquen la vulneración de otros derechos. Se recomienda que los profesionales que actúan desde los distintos ámbitos de la comunidad (socioeducativo, político, médico, judicial, etc.) reciban la formación y la orientación necesarias en materia de derechos para que sus prácticas se ajusten a los planteamientos de la CDPD. 


\section{Referencias bibliográficas}

Barnes, C. y Mercer, G. (2010): Exploring Disability (2nd Ed.). Cambridge: Polity Press.

Barr, O. et al. (2003): "Views of People with Learning Difficulties about Current and Future Accommodation: The use of focus groups to promote discussion". Disability \& Society, 18 (5): 577-597.

Beail, N. y Williams, K. (2014): "Using qualitative methods in research with people who have intellectual disabililities". Journal of Applied Research in Intellectual Disabilities, 27: 85-96.

Bloor, M. et al. (2001): Focus groups in social research. London: Sage.

Bowey, L. et al. (2005): "Assessing the Barriers to Achieving Genuine Housing Choice for Adults with a Learning Disability: The Views of Family Carers and Professionals". British Journal of Social Work, 35 (1):139-148.

Cambridge, P. y McCarthy, M. (2001): "User focus groups and Best Value in services for people with learning disabilities". Health \& Social Care in the Community, 9 (6): 476-489.

Clarkson, R. et al. (2009): "What characteristics do service users with intellectual disability value in direct support staff within residential forensic services?". Journal of Intellectual \& Developmental Disability, 34: 283-289.

Clement, T. y Bigby, C. (2012): "Competencies of front-line managers in supported accommodation: issues for practice and future research". Journal of Intellectual \& Developmental Disability, 37 (2): 131-140.

Dodevska, G. A. y Vassos, M. V. (2013): "What qualities are valued in residential direct care workers from the perspective of people with an intellectual disability and managers of accommodation services?". Journal of Intellectual Disability Research, 57 (7): 601-615.

Duggan, C. y Linehan, C. (2013): "The role of 'natural supports' in promoting independent living for people with disabilities; a review of existing literature”. British Journal of Learning Disabilities, 41 (3):199-207.

Emerson, E. y Ramcharan, P. (2010): “Models of service delivery”, en Grant, G.et al. (eds.): Learning Disability: A Life Cycle Approach (2nd Ed). Berkshire: Open University Press.

España. Real Decreto Legislativo 1/2013, de 29 de noviembre, por el que se aprueba el Texto Refundido de la Ley General de derechos de las personas con discapacidad y de su inclusión social, Boletín Oficial del Estado, 3 de diciembre de 2013, núm. 289, pp. 95635-95673.

España. Ley 39/2006, de 14 de diciembre, de Promoción de la Autonomía Personal y Atención a las personas en situación de dependencia, Boletín Oficial del Estado, 15 de diciembre de 2006, núm. 299, pp. 44142-44156.

España. Ley 13/1982, de 7 de abril, de integración social de los minusválidos, Boletín Oficial del Estado, 30 de abril de 1982, núm. 103, pp. 11106-11112.

European Commission (2009): Report of the Ad Hoc Expert Group on the transition from institutional to community-based care (en línea). <http://ec.europa.eu/social/main.jsp?langld=en\&catld=89\&newsld=614\&furtherNe ws $=$ yes $>$, acceso 23 de marzo de 2018 .

European Union Agency for Fundamental Rights (2012): Choice and control: the right to independent living. Experiences of persons with intellectual disabilities and persons with mental health problems in nine EU Member States. Luxemburgo: Publications Office of the European Union. 
Fernández Cordero, L. (2013): "El reto de la Vida Independiente. La experiencia de la fundación Síndrome de Down del País Vasco en la prestación de apoyos a Personas con Discapacidad Intelectual”. Revista Española de Discapacidad, 1 (2): 159-165.

García Iriarte, E. et al. (2016): "The role of support staff as people move from congregated settings to group homes and personalized arrangements in Ireland". Journal of Intellectual Disabilities, 20 (2):152-164.

Generalitat de Catalunya. Orden TSF/111/2017 de 7 de junio, por la que se aprueban las bases para la concesión de las ayudas de apoyo a la autonomía en el propio hogar para el año 2017, Diario Oficial de la Generalitat de Catalunya, 12 de junio de 2017, núm. 7388, pp. 1-15.

Generalitat de Catalunya. Decreto 318/2006, de 25 de julio, de servicios de acogida residencial para personas con discapacidad, Diario Oficial de la Generalitat de Catalunya, 27 de julio de 2006, núm. 4685, pp. 33802-33807.

Gómez-Vela, M. et al. (2002): "Evaluación de la calidad de vida de adultos con discapacidad intelectual en servicios residenciales comunitarios”. Revista de Psicología Aplicada, 55 (4): 591-602.

González Luna, B. (2013): “Investigando con personas con dificultades de aprendizaje”. Revista Española de Discapacidad, 1 (2): 77-94.

González Luna, B. (2010): "Promoción de la Autodeterminación en un entorno de vivienda”, en Investigación e innovación sobre Personas con Discapacidad Intelectual (en línea). <http://sid.usal.es/idocs/F8/FDO23969/ Premio_AMPANS_2010_completo.pdf>, acceso 23 de marzo de 2018.

Guest, G. et al. (2012): Applied Thematic Analysis. Londres: Sage.

Hatton, C. et al. (2009): "Developing measures of job performance for support staff in housing services for people with intelectual disabilities". Journal of Applied Research in Intellectual Disabilities, 22 (1): 54-64.

Haya, I. et al. (2014): "Observaciones metodológicas sobre la investigación inclusiva: 'Me gustaría que sacarais que la persona con discapacidad tiene su propio pensamiento'”. Revista de investigación en educación, 12 (2):135-144.

Hawkins, R. et al. (2011): "Duty of care and autonomy: how support workers managed the tension between protecting service users from risk and promoting their independence in a specialist group home". Journal of Intellectual Disability Research: JIDR, 55 (9): 873-884.

Hollomotz, A. (2017): "Successful interviews with people with intellectual disability". Qualitative Research, 18 (2): 153-170.

Hollomotz, A. y The Speakup Committee (2009): "May we please have sex tonight?" - people with learning difficulties pursuing privacy in residential group settings". British Journal of Learning Disabilities, 37 (2): 91-97.

Huete, A. et al. (2016): Jóvenes con discapacidad en España 2016. Madrid: Injuve-CERMI.

Huete, A. y Quezada, M. Y. (2014): "Análisis de Retorno Social de la Inversión en dos sistemas de apoyo a personas con gran discapacidad: asistencia personal y servicio residencial”. Revista Española de Discapacidad, 2 (1): 51-69.

Illán, N. y Molina, J. (2013): "La Filosofía de Escuela de vida. Sus aportes para la promoción de la vida independiente en el colectivo de personas con discapacidad intellectual”. Revista Latinoamericana de Educación Inclusiva, 7: 23-38.

Kaehne, A. y O'Connell, C. O. (2010): "Focus groups with people with learning disabilities". Journal of Intellectual Disabilities, 14 (2): 133-145. 
Kilbane, J. y McLean, T. (2008): “Exploring the history of person centred practice”, en Thompson, J. et al. (eds.): Person Centred Practice for Professionals. Inglaterra: Open University Press.

Klein, J. (2000): Ayudar a las personas con discapacidad a vivir en un hogar propio. Barcelona: FCSD.

Latorre, C. y Liesa, M. (2016): "La inclusión social de personas con diversidad funcional en una experiencia de Vida Independiente". Revista de Educación Inclusiva, 9 (2):171-185.

Liesa, M. y Vived, E. (2010): "Discapacidad, edad adulta y vida independiente. Un estudio de casos". Educación y Diversidad, 4 (1): 101-124.

Mansell, J., et al. (2010): "Deinstitutionalisation and community living: position statement of the Comparative Policy and Practice Special Interest Research Group of the International Association for the Scientific Study of Intellectual Disabilities". Journal of Intellectual Disability Research, 54 (2):104-112.

McConkey, R. et al. (2016): "Relocating people with intellectual disability to new accommodation and support settings: Contrasts between personalized arrangements and group home placements". Journal of Intellectual Disabilities, 20 (2): 109-120.

McConkey, R. y Collins, S. (2010): "The role of support staff in promoting the social inclusion of persons with an intellectual disability”. Journal of Intellectual Disability Research, 54 (8): 691-700.

Naciones Unidas (2006): Convención Internacional sobre los Derechos de las Personas con Discapacidad. Nueva York: ONU.

Navallas, M. y Verdugo, M. A. (2009): "Integrando calidad de vida y calidad de gestión en un servicio de viviendas y residencia para personas con discapacidad intelectual”. Siglo Cero. Revista Española sobre Discapacidad Intelectual. 40 (2): 5-22.

Puyaltó, C. (2016): La vida independiente de las personas con discapacidad intelectual. Análisis de los apoyos y las barreras que inciden en la consecución de sus proyectos de vida (tesis doctoral). Universidad de Girona.

Roeden, J. M. et al. (2011): "The Views of Clients with Mild Intellectual Disabilities Regarding their Working Relationships with Caregivers”. Journal of Applied Research in Intellectual Disabilities, 24 (5): 398-406.

Rojas, S. (2008): “La 'voz' de las personas con discapacidad intelectual en investigación educativa. Repensando las prácticas de investigación”. Revista de Educación, 345: 377-398.

Rojas, S. (2004): Autodeterminación y calidad de vida en personas discapacitadas. Experiencias desde un hogar de grupo (tesis doctoral). Universidad Autónoma de Barcelona.

Ruf, J. (2006): "El servicio de apoyo en el propio hogar, perspectivas iniciales de una nueva modalidad de servicio de apoyo a la persona". Revista de Educación Social, 5 (en línea). <http://www.eduso.net/res/?b=8\&c=68\&n=192>, acceso 23 de marzo de 2018.

Ruiz, S. (2016): La figura del Asistente Personal en la promoción de la autonomía y la vida independiente de las personas con discapacidad intellectual (tesis doctoral). Universidad Autónoma de Madrid.

Saldaña, J. (2013): The Coding manual for Qualitative Researchers. Thousand Oaks: Sage.

Seidman, I. (2006): Interviewing as a qualitative research. A guide for researchers in education and the social sciences. Nueva York y Londres: Teachers College, Columbia University.

Shogren, K. A y Turnbull, R. (2014): "Core concepts of disability policy, the Convention on the Rights of Persons with Disabilities, and public policy research with respect to developmental disabilities". Journal of Policy and Practice in Intellectual Disabilities, 11 (1): 19-26. 
Susinos, T. y Parrilla, A. (2013). "Investigación inclusiva en tiempos difíciles. Certezas provisionales y debates pendientes". Revista Electrónica Iberoamericana sobre Calidad, Eficacia y Cambio en Educación, 11 (2): 88-98.

Susinos, T. y Parrilla, A. (2008): "Dar la voz en la investigación inclusiva. Debates sobre inclusión y exclusión desde un enfoque biográfico-narrativo". Revista Electrónica Iberoamericana sobre Calidad, Eficacia y Cambio en Educación, 6 (2): 157-171.

Tøssebro J. et al. (2012): "Normalization fifty years beyond- Current trends in the Nordic countries". Journal of Policy and Practice in Intellectual Disabilities, 9 (2): 134-146.

Tracy, S. J. (2010): “Qualitative Quality: Eight ‘Big-Tent' Criteria for Excellent Qualitative Research”. Qualitative Inquiry, 16 (10): 837-851.

Verdugo, M. A. et al. (2014): Escala San Martín: Evaluación de la calidad de vida de personas con discapacidades significativas. Santander: Fundación Obra San Martín, INICO.

Verdugo, M. A. et al. (2013): Escala INICO-FEAPS. Evaluación integral de la Calidad de Vida de personas con discapacidad intelectual o del desarrollo. Salamanca: INICO.

Verdugo, M. A. et al. (2009a): ANED country report on the implementation of policies supporting independent living for disabled people (en línea). <https://www.cedd.net/es/documentacion/catalogo/Record/168761>, acceso 23 de marzo de 2018.

Verdugo, M. A. et al. (2009b): Escala de Calidad de vida - GENCAT. Barcelona: ICASS, Generalitat de Catalunya.

Vived, E. et al. (2013): "Avanzando hacia la vida independiente: planteamientos educativos en jóvenes con discapacidad intelectual”. Revista Española de Discapacidad, 1 (1): 119-138.

Wahlström, L. et al. (2014): "Promoting health of people with intellectual disabilities: Views of professionals working in group homes”. Journal of Intellectual Disabilities, 18 (2): 113-128. 